LHCb early running experience

This article has been downloaded from IOPscience. Please scroll down to see the full text article. 2010 JINST 5 C12022

(http://iopscience.iop.org/1748-0221/5/12/C12022)

View the table of contents for this issue, or go to the journal homepage for more

Download details:

IP Address: 137.138.124.142

The article was downloaded on 11/07/2011 at 10:46

Please note that terms and conditions apply. 
Topical Workshop on Electronics for Particle Physics 2010, 20-24 SEPTEMBER 2010, Aachen, Germany

\title{
LHCb early running experience
}

\author{
K. Hennessy ${ }^{1}$ \\ Department of Physics, University of Liverpool, \\ Oliver Lodge Laboratory, Liverpool L69 7ZE, United Kingdom \\ E-mail: karol.hennessy@cern.ch
}

ABSTRACT: The LHC 7TeV Physics programme started at the end of March 2010. This paper highlights the experiences of running the LHCb detector with early pp-collisions from the LHC. An overview of the operation of the detector with the first $1 p b^{-1}$ will be given, and the challenge of running the detector smoothly in the initial data taking stages. Focus will be given to the performance of the hardware and the interplay between LHCb and the machine. We highlight our successes, and report on progress made in solving outstanding sub-detector issues.

KEYWORDS: Large detector systems for particle and astroparticle physics; Particle tracking detectors; Detector control systems (detector and experiment monitoring and slow-control systems, architecture, hardware, algorithms, databases); Cherenkov detectors

\footnotetext{
${ }^{1}$ On behalf of the $\mathrm{LHCb}$ collaboration
} 


\section{Contents}

1 Introduction 1

2 Sub-detector performance 2

3 General detector performance $\quad 4$

4 Conclusion 5

\section{Introduction}

The Large Hadron Collider Beauty detector [2] (figure 1) is a flavour physics detector, designed to detect decays of b- and c-hadrons for the study of $\mathrm{CP}$ violation and rare decays. In pp collisions at LHC [1] energies, $b \bar{b}$ production is primarily in the forward/backward direction. LHCb has been designed as a forward arm spectrometer, to exploit this fact. LHCb is a precision experiment and will reach its nominal luminosity in $2011-L=2 \times 10^{32} \mathrm{~cm}^{-2} \mathrm{~s}^{-1}$, and is expected to operate in current configuration to 2015. Due to the intense radiation environment of the LHC, nearly all of LHCb's components are required to be radiation hard for ten years of operation at nominal luminosity.

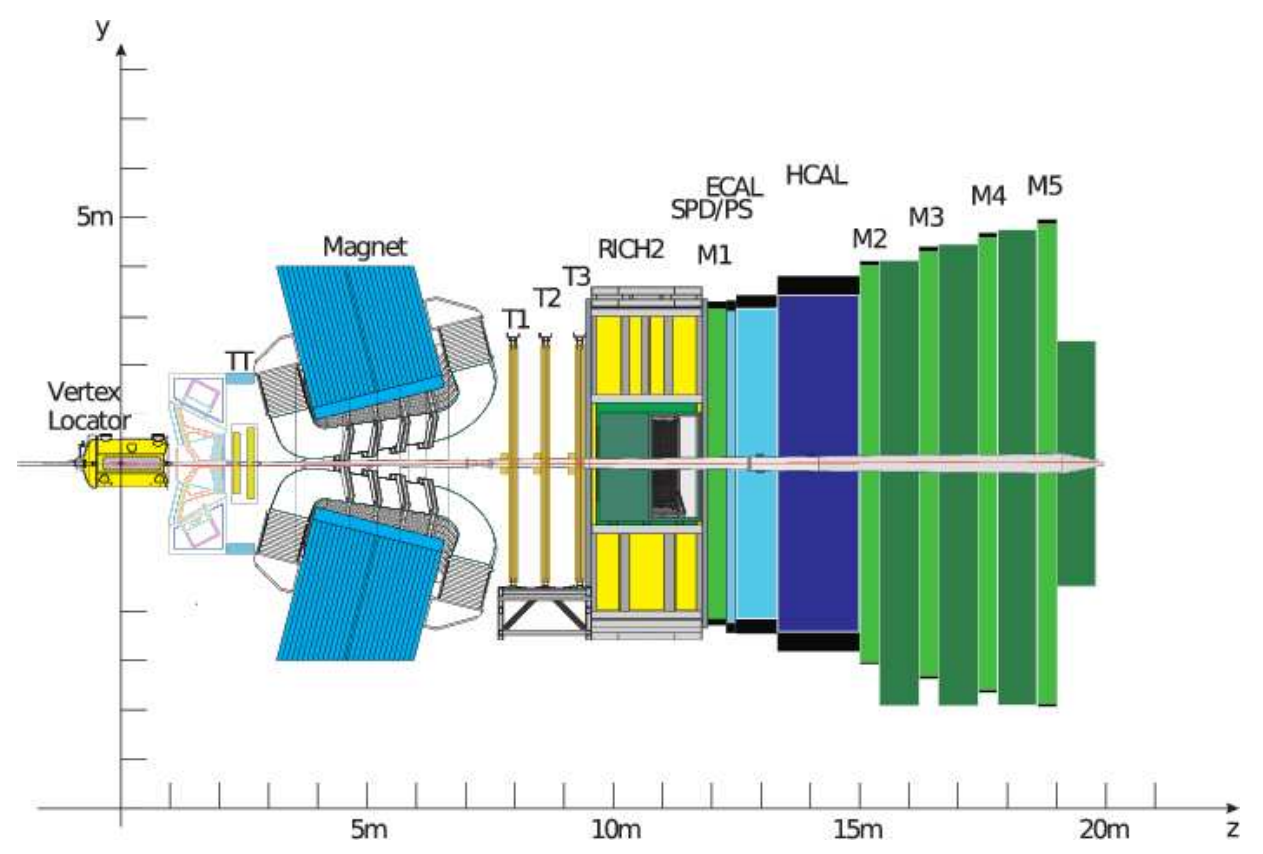

Figure 1. The LHCb detector - starting at the interaction point and proceeding downstream, we have the Vertex Locator, RICH1, TT, Magnet, T1-T2-T3 tracking stations, RICH2, Calorimeters and finally Muon chambers. 
The tracking system for $\mathrm{LHCb}$ consists of a vertex detector, a dipole magnet and a series of tracking stations. The Vertex Locator (VELO) detector is a silicon strip detector surrounding the interaction point at LHCb. The sensors are made of $n^{+}-i n-n$ silicon, and are positioned only $8 \mathrm{~mm}$ from the beam line during data taking. The VELO is split into two halves such that they can be retracted to $29 \mathrm{~mm}$ when the beam is considered less stable during LHC injection. The VELO is required to have excellent impact parameter resolution, essential for the reconstruction of B decays.

The silicon tracker comprises two sub-detectors using p-in-in silicon technology. These are the Tracker Turicensis (before the magnet) and the Inner Tracker (after the magnet). The Outer Tracker is a straw tracker surrounding the Inner Tracker. It uses $\mathrm{CO}_{2}$-Argon gas mixture, and it has a fast $<50$ ns drift time.

The particle ID system consists of Čerenkov detectors, a calorimeter system, and a muon detector. LHCb has two Čerenkov detectors, RICH1 and RICH2 used for hadron separation. RICH 1 which is upstream of the dipole magnet uses and a Aerogel and $C_{4} F_{10}$ for the identification of low momentum particles $(\mathrm{p}<60 \mathrm{GeV} / \mathrm{c})$. RICH2 is downstream of the magnet and uses $\mathrm{CF}_{4}$ to identify high momentum particles $(\mathrm{p}<100 \mathrm{GeV} / \mathrm{c})$. RICH uses Hybrid Photon Detectors to record the Čerenkov photons produced inside the gas.

The Calorimeter system is a typical electromagnetic calorimeter (ECAL) and a hadronic calorimeter (HCAL) combination. The calorimeters provide measurements of high- $E_{T}$ photons, electrons and hadrons to the trigger system. The calorimeters are typical calorimeters consisting of interleaved scintillator and absorber. Each scintillator tile is read out via MAPMT pixel photomultipliers.

The muon stations are responsible for muon ID and providing muon $P_{T}$ measurements to the trigger. The muon system is comprised of five stations (M1-M5) of Multiwire Proportional Chambers (MWPCs). M2-M5 are located furthest from the interaction point, and the first station, M1, is situated between RICH1 and the Calorimeters. The innermost section of the first muon chamber uses triple GEM detectors rather than MWPCs to better cope with the higher particle fluences in this region.

LHCb has one hardware trigger, L0, and a software trigger HLT, which runs on an event filter farm. L0 takes energy and momentum information from the calorimeters, and muon stations at $40 \mathrm{MHz}$ input rate. This information is fed over $1.6 \mathrm{GHz}$ high speed opto-links to the L0 Decision Unit. The L0 Decision Unit selects events at $1 \mathrm{MHz}$ readout rate.

\section{Sub-detector performance}

The start of data taking with $3.5 \mathrm{TeV}$ beams provided an essential commissioning period for LHCb. This was the first period that the VELO was operated fully closed with beam. The first full operation of closing procedure was performed with due care and scrutiny. Since then, several improvements in the automation of the procedure and monitoring of the beam position have been made. The current operation takes less than five minutes. The VELO shows excellent S/N of approximately 20:1 and a hit resolution of $4 \mu \mathrm{m}$ at its peak, notably the best resolution at the LHC. This has enabled the VELO to provide essential feedback to LHC with respect to the behaviour of the beams. It has been used to extract beam shapes and monitor stability of beam during the earliest stages of data taking. 
The TT and IT have shown resolutions of $65 \mu \mathrm{m}$ and their S/N agrees well with expectation, and both have a noise occupancy of less than $10^{-5}$. Some repairs and replacements will take place in the winter technical stop (2010). These include new hybrids to replace broken wire bonds on TT modules.

However, largest cause of inefficiency in the silicon trackers currently is due to the VCSEL ${ }^{1}$ failures. These are front-end optical transmitters used in the silicon trackers and many of the other sub-detectors in LHCb. Approximately two VCSELs per month are failing at the current rate. Up to the end of August 2010, a total of sixteen VCSELs failed in LHCb. The reason for the failures is, as yet, unknown but spares have been made available Similar VCSEL failures have been seen in ATLAS, and a cross-detector team is in contact the manufacturer, and both are performing tests to investigate the problem. An advantage $\mathrm{LHCb}$ has, as an open detector, is that it is easier to replace these components than in a closed detector such as ATLAS.

The Front-end ASIC for the VELO and ST is the Beetle chip [3]. The chip reads out at $1 \mathrm{MHz}$ and can provide analogue or digital output. For LHCb, the signals are digitised off-chip. In VELO for example, the analogue signals are sent over $60 \mathrm{~m}$ differential analogue copper cables to the counting room for digitisation with essentially no loss of $\mathrm{S} / \mathrm{N}$. Some minor issues have been noticed with the Beetle chip. A pedestal (or voltage baseline) shift has been observed on certain chips at different trigger rates. The pedestal shifts have not been seen as continuously varying with rate but rather as a step function at approximately $10 \mathrm{kHz}$. In order to deal with this effect, separate pedestals have been calculated when running at a low $(<10 \mathrm{kHz})$ and high $(>10 \mathrm{kHz})$ trigger rate. The Beetle has also shown some derandomiser problems. The Beetle should have a 16 deep derandomiser, however, when operating at high rate $(>200 \mathrm{kHz})$ dead time has been observed when the derandomiser is more than half full. An emulator has been put in place in the trigger logic of $\mathrm{LHCb}$ to compensate for this effect.

For the RICH detectors, Hybrid Photon Detectors (HPDs) have been employed to read out the Čerenkov photons produced in the RICH gases. The HPDs have the advantage of being noise-less, in that they can detect single photons. The LHCBPIX1 pixel chip forms part of the readout and the anode assembly of the HPD. A design error in the pixel chip introduces small dead time at high rate, such that no consecutive event readout is possible. A fraction of the HPDs have failed. Approximately, half of these failures are due to the aforementioned VCSEL problem, and the rest are due to the HPDs themselves. These will be replaced in the winter technical stop.

The TELL1 [4] is a common readout board for LHCb and is used to read out all of the frontends in LHCb with the exception of the RICH detectors. It can take both analogue or digital input. Five FPGAs are used to process and packetise the data for output on the gigabit ethernet interface. Specific TELL1 firmware has been developed for each sub-detector's data processing needs. Thus far this year, ten out of a total of 292 TELL1s have had performance problems deeming their replacement, although the rate of failure has decreased in recent months. The failures are often coincident with general powercuts. The firmware on the FPGAs enables the same board to be used by different sub-detectors, however, this requires a lot of testing/emulation before each firmware upgrade can be qualified. As a result, this requires manpower support from the sub-detectors.

Overall, the sub-detectors of LHCb have shown excellent performance, with most showing over $99 \%$ of the readout channels operational (table 1).

\footnotetext{
${ }^{1}$ VCSEL - Vertical-cavity surface-emitting laser.
} 
Table 1. Sub-detector operational efficiency.

\begin{tabular}{|c|c|}
\hline Sub-detector & Efficiency (\%) \\
\hline VELO & 99.3 \\
TT & 99.8 \\
Inner Tracker & 98.6 \\
Outer Tracker & $>99.9$ \\
RICH & 96.3 \\
Calorimeters & $>99.9$ \\
Muon Detector & 99.8 \\
\hline
\end{tabular}

\section{General detector performance}

One of the most essential operations for detector performance is to accurately time the detector to the LHC beam crossings. To this end, the early data taking period was used to take calibration data necessary for this task. The global LHCb timing is performed by timing the calorimeter system to the beam crossings. The Calorimeter timing resolution is approximately 1ns [5]. Each of the other sub-detectors are subsequently timed to the calorimeters. Overall, each of the sub-detectors have a time resolution of approximately $\sim 2-6$ ns or less [6-8].

Many improvements have been made on reducing down-time during beam. Such improvements include automatic recovery of failing DAQ systems, and the possibility to exclude/include trigger farms rapidly during data taking. Settings have been optimised for running at high rate ( $\sim 200 \mathrm{kHz}$ L0 at time of writing). In terms of the interface with LHC: the interlocking system has been checked rigorously and extra levels of machine state checking have been put in place to protect sensitive parts of the detector. The LHCb Shift Leader is required for confirmation of action as the detector switches between safe and non-safe states.

As more has been learned about the LHC, the rate of collisions (luminosity) has increased by several orders of magnitude. In effect, this means that the operating parameters for the detectors on the LHC ring are constantly changing. With the very first data, where only one bunch in each beam was colliding the data rate was low enough to capture every event, and the detector effectively ran with a pass-through trigger. As more bunches were added to the machine, the data rate increased and it was necessary to employ the trigger system. Figure 2 shows the integrated luminosity delivered to, and recorded by $\mathrm{LHCb}$ this year. $\mathrm{LHCb}$ has shown over $90 \%$ uptime during data taking, and during recent long runs this figure is typically above $97 \%$.

The LHC has also squeezed the beams such that more than one proton-proton interaction can occur in each bunch crossing, with the current average being 2.0 interactions per bunch crossing at the time of writing. LHCb was originally designed to operate with an average of 0.7 events per bunch crossing [9]. This has also increased the data rate and event size (from $35 \mathrm{kB}$ to $85 \mathrm{kB}$ ). Software and firmware have been adapted to cope with larger event sizes. Thus one can conclude that $\mathrm{LHCb}$ is currently operating beyond its original design. 


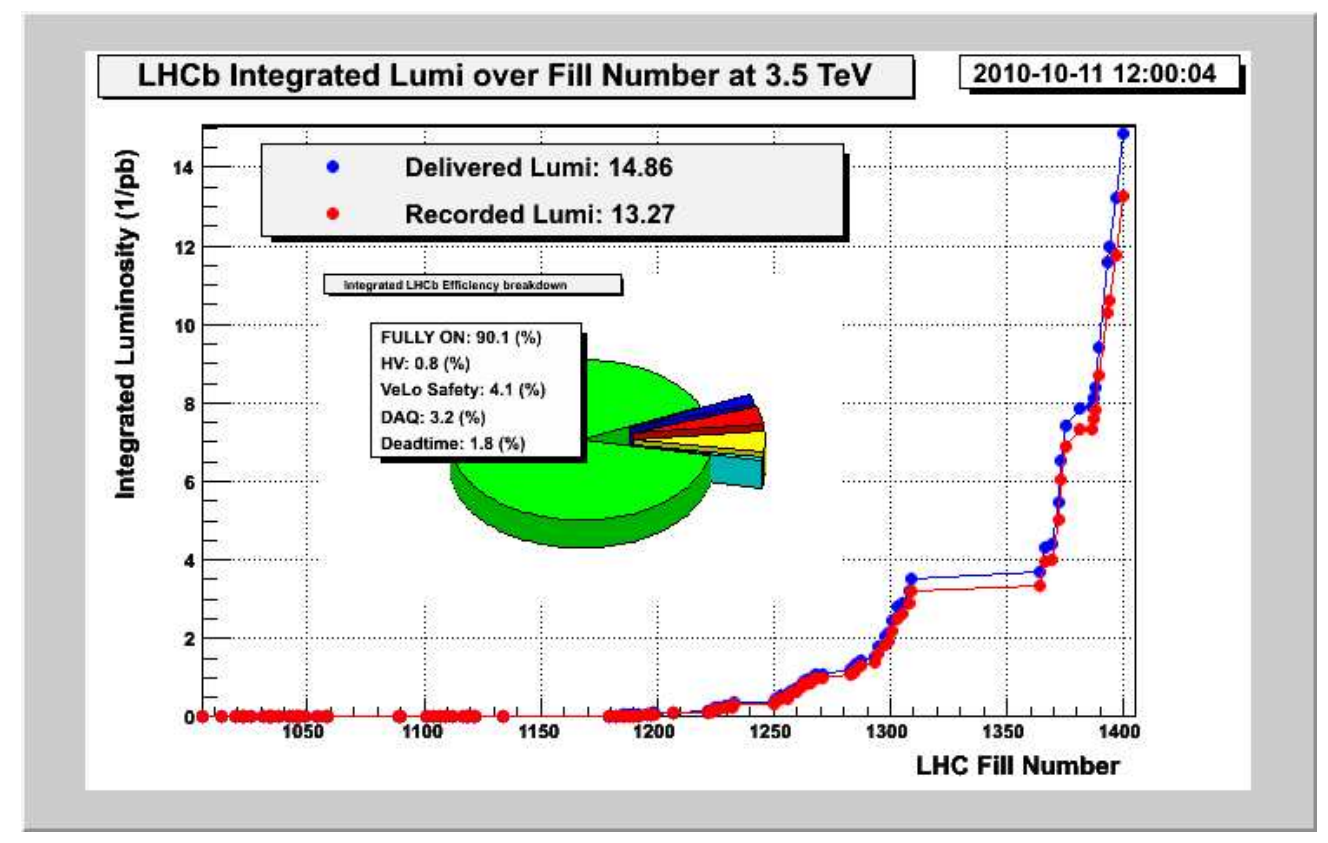

Figure 2. LHCb Integrated Luminosity.

\section{Conclusion}

LHCb has shown over $90 \%$ uptime for total integrated luminosity delivered this year. Typically, in recent runs this has been above $97 \%$. Most sub-detectors have greater than $99 \%$ working channels, and others will be fixed/replaced in winter technical stop. LHCb is a precision physics detector with unique capability and will reach its physics potential next year.

\section{References}

[1] L. Evans and P. Bryant (eds.), LHC Machine, 2008 JINST 3 S08001.

[2] The LHCb collaboration, The LHCb Detector at the LHC, 2008 JINST 3 S08005.

[3] S. Loechner and M. Schmelling, Beetle Reference Manual, LHCb-2005-105.

[4] G. Haefeli et al., The LHCb DAQ interface board TELL1, Nucl. Instrum. Meth. A 560 (2006) 494.

[5] M. Calvo Gomez, Time Alignment and Calibration of the LHCb Calorimeter, LHCb-CONF-2010-007.

[6] M. Needham et al., Fine time alignment of the LHCb Inner Tracker with the LHC injection tests, LHCb-PUB-2009-020.

[7] I. Mous, Timing of the LHCb VELO, LHCb-PUB-2010-006.

[8] M. Frosini et al., Measurement of the time alignment between muon detector and calorimeters with the 2008 cosmic runs, LHCb-PUB-2009-028.

[9] The LHCb collaboration, LHCb Technical proposal, CERN-LHCC-98-004. 